\title{
NORMAS INTERNACIONALES DE INFORMACIÓN FINANCIERA (NIIF 9), MEDICIÓN Y CLASIFICACIÓN DE LOS INSTRUMENTOS FINANCIEROS
}

\author{
INTERNATIONAL FINANCIAL REPORTING STANDARDS (IFRS 9), \\ MEASUREMENT AND CLASSIFICATION OF FINANCIAL \\ INSTRUMENTS
}

\section{Erika Sonia Quiñonez Alvarado', Mariana de Jesús Lozano Robles², José Luis Romero Villagrán ${ }^{3}$}

\section{1,2,3 - Universidad de Guayaquil, Guayaquil, Ecuador}

1. Email: erika.quinoneza@ug.edu.ec ORCID: $\underline{\text { https://orcid.org/0000-0002-3520-5183 }}$

2. Email: mariana.lozanorob@ug.edu.ec ORCID: https://orcid.org/0000-0001-6288-5340

3. Email: jose.romerov@ug.edu.ec ORCID: https://orcid.org/0000-0002-0593-3905

Recibido: 11/05/2021 Aceptado: 21/07/2021

Para Citar: Quiñonez Alvarado, E. S., Mariana de Jesús Lozano Robles, M. de J. L. R., \& José Luis Romero Villagrán, J. L. R. V. (2021). Normas Internacionales de Información Financiera (NIIF 9), medición y clasificación de los instrumentos financieros. Revista Publicando, 8(31), 194-206. https://doi.org/10.51528/rp.vol8.id2244

\begin{abstract}
Resumen:
El objetivo general de este artículo es analizar la Norma Internacional de Información Financiera (NIIF 9) evaluando su clasificación y medición de instrumentos financieros. La metodología utilizada se basa en un diseño bibliográfico de tipo documental. Como conclusión se puede mencionar que las Normas Internacionales de Información financiera, son fundamentales para lograr una mayor transparencia en la presentación de los estados financieros y en el caso más específico la NIIF 9 permite estudiar los instrumentos financieros, medirlos y evaluarlos teniendo una mayor claridad del comportamiento de cada uno de ellos, lo que permite tomar decisiones más precisas. Es evidente que la NIF 9 es compleja, sin embargo puede ser analizada y comprendida para la aplicación de su fundamentación logrando tener un impacto en el análisis de los instrumentos financieros y su medición. La NIIF 9 al igual que el resto de las normas se puede ir adaptando de acuerdo a los cambios producto de la globalización y las exigencias del mercado, de esta forma se mantiene un equilibrio económico en cada una de las empresas que deban aplicar estas normas y se evita sucumbir en errores que sean desencadenantes de malos procedimientos financieros y comerciales.
\end{abstract}

Palabras clave: Instrumentos financieros, NIIF 9, Estados financieros.

\begin{abstract}
:
The general objective of this article is to analyze the International Financial Reporting Standard (IFRS 9) by evaluating its classification and measurement of financial instruments. The methodology used is based on a documentary bibliographic design. As a conclusion it can be mentioned that the International Financial Reporting Standards are fundamental to achieve greater transparency in the presentation of financial statements and in the more specific case IFRS 9 allows studying financial instruments, measuring and evaluating them having a greater clarity of the behavior of each one of them, which allows making more accurate decisions. It is evident that IFRS 9 is complex, however it can be analyzed and understood for the application of its basis, achieving an impact on the analysis of financial instruments and their measurement. IFRS 9 , as well as the rest of the standards, can be adapted according to the changes resulting from globalization and market requirements, thus maintaining an economic balance in each of the companies that must apply these standards and avoiding succumbing to errors that may trigger bad financial and commercial procedures.
\end{abstract}

Keywords: Financial instruments, IFRS 9, Financial statements. 


\section{INTRODUCCIÓN}

$\mathrm{P}$ roducto de la globalización, el mundo cambia constantemente en todos sus aspectos, evolucionando y adaptándose a nuevas situaciones, la sociedad y los gobiernos son eslabones importantes pues son los encargados de tomar decisiones personales o a nivel de cualquier organización con respecto a la economía y el aspecto financiero lo que ha generado grandes avances en cuanto a su regulación con el surgimientos de nuevas normativas por las cuales deben regirse. En este sentido se mencionan las Normas Internacionales de Información Financiera (NIIF, o, «IFRS» por sus siglas en inglés) que juegan un papel preponderante en la búsqueda de estándares y beneficios para las empresas.

Esto lo refuerza Durán et al. (2017), cuando manifiestan que es de gran importancia que los gerentes y administradores afronten la necesidad de poseer conocimientos de alto nivel que les permitan tomar decisiones oportunas, ello requiere la utilización de instrumentos adecuados desde el punto de vista financiero para administrar eficientemente sus empresas y alcanzar los objetivos establecidos.

Parte de estos instrumentos esenciales son las NIIF, y producto de los cambios generados por diversos factores en los últimos tiempos. Algunas NIIF han sido reformadas y entraron en vigor entre el año 2018 y 2019, estas reformas fueron emitidas por el International Accounting Standards Board (IASB), y estas son las NIIF 9, NIIF 15 y NIIF 16 que impactan directamente en los estados financieros de las empresas (MoralesDíaz, 2018).
Para el estudio de este artículo nos enfocaremos en el análisis de la NIIF 9 o IFRS 9 (por sus siglas en inglés) que implicara nuevos desafíos para las entidades financieras en cuanto a la clasificación y medición de los activos financieros y como indica Rodríguez (2017), esta sustituye a la NIC 39 y a todas las versiones anteriores de la NIIF 9, e incorpora importantes modificaciones a la clasificación de instrumentos financieros, que impactan no solamente el área contable sino todas las áreas involucradas en el modelo de negocio de las empresas.

Las NIIF 9 entró en vigencia el 01 de enero de 2018 y fue ejecutadas en tres fases: en primer lugar estuvo dirigida a la clasificación y medición, seguidamente a la medición de deterioro, y en tercer lugar se enfocó a la contabilidad de coberturas teniendo un gran impacto en los estados financieros de las empresas prestadoras de créditos (Parrales y Castillo, 2018). Por lo tanto las empresas a nivel mundial deben mantenerse al día con las diferentes modificaciones de dicha norma a fin de poder comprender y adaptarse a sus exigencias.

El objetivo general de esta investigación se basa en analizar la Norma Internacional de Información Financiera (NIIF 9), evaluando su clasificación y medición de instrumentos financieros con el propósito de conocer con mayor profundidad el impacto que tiene sobre las decisiones empresariales. La metodología utilizada en este artículo se basa en un diseño bibliográfico de tipo documental.

\section{METODOLOGÍA}

Esta investigación se realizó basándose en un diseño bibliográfico de tipo documental. El trabajo 
se fundamenta en la revisión sistemática, rigurosa y profunda de material documental de cualquier clase, donde se efectúa un proceso de abstracción científica, generalizando sobre la base de lo fundamental, partiendo de forma ordenada y con objetivos precisos (Palella y Martins, 2010). La investigación documental se concreta exclusivamente en la recopilación de información de diversas fuentes, con el objeto de organizarla describirla e interpretarla de acuerdo con ciertos procedimientos que garanticen confiabilidad y objetividad en la presentación de los resultados (Palella y Martins, 2010). Para lograr este propósito se utilizaron herramientas como textos, documentos y artículos científicos publicados disponibles en la web.

Los objetivos de esta investigación se basaron en, Identificar el impacto de las NIIF 9 sobre los instrumentos financieros, de igual manera se pretende analizar la clasificación de estos instrumentos financieros y la influencia que tienen en el desenvolvimiento de las empresas.

\section{RESULTADOS Y DISCUSIÓN \\ Las NIIF y su importancia}

Las Normas Internacionales de Información Financiera (NIIF), son un conglomerado de reglas o pautas a seguir en el proceso contable y que los países y empresas han tenido que adaptar y reformar algunos procedimientos ya que son bien aceptadas en el mundo. En este sentido su importancia según Ortegón (2017), es que mejora el desarrollo confiable, transparente y efectivo de las diversas transacciones internaconales, esto con el propósito de que las empresas logren un crecimiento económico que trascienda hasta un nivel internacional.
También se puede mencionar que las Normas Internacionales de Información Financiera (NIIF) son normas elaboradas con el fin de desarrollar una normativa clara y uniforme referente a las normas de valoración aplicables a cuentas anuales y consolidadas de determinadas formas sociales, bancos y otras entidades financieras. Estas están diseñadas pensando en empresas grandes, con relevancia en el entorno económico y proyección internacional. Estas normas son obligatorias en la Unión Europea pero opcionales para uso personal de cualquier empresa, y son ideales para empresas que realizan negocios internacionales (Rodríguez, 2015).

En este sentido se menciona un listado de las NIIF vigentes y aceptadas por gran parte del mundo y vigentes en Ecuador:

- $\quad$ NIIF 1: Adopción por primera vez de las NIIF

- NIIF 2: Pagos basados en acciones

- $\quad$ NIIF 3: Combinación de negocios

- $\quad$ NIIF 4: Contratos de seguro

- $\quad$ NIIF 5: Activos no corrientes mantenidos para la venta y operaciones descontinuadas

- NIIF 6: Exploraciñon y evaluación de recursos minerales

- NIIF 7: Instrumentos financiieros: información a revelar

- $\quad$ NIIF 8: Segmentos de operación

- $\quad$ NIIF 9 Instrumentos financieros

- NIIF 10: Estados financieros consolidados

- NIIF 11: Acuerdos conjuntos

- NIIF 12: Información a revelar sobre 
participaciones en otras entidades

- NIIF 13 Medicion del valor razonable

- NIIF 14: Cuentas de diferimientos de actividades reguladas

- NIIF 15: Ingresos de actividades ordinarias procedentes de contratos con clientes

- NIIF 16: Arrendamientos (Enríquez, 2019)

\section{La NIIF 9: aspectos relevantes}

La NIIF 9 fue creada debido a la crisis presentada en el año 2008 donde fue evidente la debilidad existente en la información financiera que presentaban las empresas para poder enfrentar crisis económicas de alta envergadura. En este sentido numerosos organismos incluido el G20 indicaron que existía la necesidad de mejorar y reforzar las fallas presentes para mejorar los estándares de presentación de información en los estados financieros pues el objetivo de los mismos es revelar información referente al desempeño financiero y de los flujos de efectivo de las empresas que permiten tomar decisiones importantes (Medina, 2018).

Esta norma viene a sustituir a la NIC 39 con algunas excepciones y a todas las NIIF 9 anteriormente establecidas por la IASB, su primera versión fue publicada en el año 2009 y se realizaron diversas modificaciones a finales del año 2010 y en 2013 y finalmente se completo en 2014 entrando en vigencia en el año 2018 (Medina, 2018). Su principal objetivo es:

Establecer principios sobre el reconocimiento y valoración de activos y pasivos financieros, para que la información de los estados financieros sea útil y relevante para sus usuarios, para permitirles evaluar las cantidades, momento del tiempo e incertidumbre de los futuros flujos de caja de la entidad (Medina, 2018, pág. 17).

Instrumentos financieros y su clasificación.

La Junta de Normas Internacionales de Contabilidad (2013) citado por Goyburo (2018), definen los Instrumentos financieros como cualquier contrato que dé lugar a un activo financiero en una entidad y a un pasivo financiero o a un instrumento de patrimonio en otra entidad.

Asimismo, Buján (2018) define los instrumentos financieros como aquellos que estan representados por el efectivo, el derecho de propiedad sobre una entidad, o por un derecho contractual de recibir o entregar, efectivo u otro instrumento financiero. En la Tabla 1 (siguiente página) se indican algunos ejemplos de instrumentos financieros.

Los instrumentos financieros presentan una serie de aspectos comunes que se deben conocer para poder evitar errores al momento de clasificarlos y estos aspectos son mostrados a continuación.

Aspectos a considerar para clasificar los instrumentos financieros:

- Se necesita un contrato, por lo que aquellos activos y pasivos cuyo origen no sea contractual, y que, por ejemplo, nacen como resultado de requisitos gubernamentales, como ingresos de impuestos, dejan de ser activos o pasivos

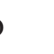


Tabla 1

Ejemplos de instrumentos financieros.

\section{ACTIVO FINANCIERO PASIVO FINANCIERO}

\section{INSTRUMENTO DE PATRIMONIO}

1.- Efectivo

2.-Un instrumento de patrimonio de otra entidad.

3.-Un derecho contractual: como recibir efectivo u otro instrumento; o a intercambiar activos o pasivos financieros con otra entidad en condiciones favorables para la entidad.

4.-Un contrato que será o podrá ser liquidado utilizando instrumentos de patrimonio propio de la entidad
1.-Una obligación

contractual: como entregar

efectivo u otro instrumento; o a intercambiar activos o pasivos financieros con otra entidad en condiciones favorables para la entidad.

2.-Un contrato que será o podrá ser liquidado utilizando instrumentos de patrimonio propio de la entidad, y sea: un instrumento no derivado, o un instrumento derivado que será o podrá ser liquidado mediante una forma distinta al intercambio de un importe fijo de efectivo, o de otro activo financiero, por una cantidad fija de los instrumentos de patrimonio propio de la entidad.

Fuente: (Goyburo, 2018).

financieros porque no son contractuales. En este sentido todos aquellos pasivos y activos financieros que tengan un origen contractual son considerados instrumentos financieros.

- Los prestamos de acuerdo con la NIC 17, son un derecho de cobro para una parte y una obligación de pago para la otra. De ellos nace activos (derecho de cobro) y pasivos financieros (obligación de pago) cuyo devengo no suele coincidir con el momento de su cobro o pago.

- Activos tangibles (existencias, inmuebles), intangibles (patente, fondo de 
comercio) y aquellos procedentes de un préstamo, no se consideran activos financieros (Medina, 2018).

- Pago de bienes y servicios: la compraventa de bienes o servicios a crédito da lugar al nacimiento de un activo o pasivo financiero cuando los bienes se transfieren.

Comparativo entre la NIC 39 y la NIIF 9

Filgueira (2017), menciona que:
El IASB ha pretendido siempre que la NIIF 9 sustituya a la NIC 39 en su totalidad. Sin embargo, en respuesta a las solicitudes de las partes interesadas de que la contabilidad de instrumentos financieros debía mejorarse con rapidez, el IASB dividió su proyecto para sustituir la NIC 39 en tres fases principales. A la medida que el IASB completo cada fase, creo capítulo en la NIIF 9 que sustituyeron los requerimientos correspondientes de la NIC 39 (Filgueira, 2017).

De esta manera Rodríguez (2017), establece un cuadro comparativo que indica los temas donde surgen cambios entre ambas normas y se muestra en la Tabla 2.

Tabla 2

Comparatición entre la NIC 39 y la NIIF 9.

\begin{tabular}{|c|c|c|c|}
\hline TEMA & CRITERIO & NIC 39 & NIIF 9 \\
\hline $\begin{array}{l}\text { Parámetro para la } \\
\text { clasificación de } \\
\text { instrumentos }\end{array}$ & & $\begin{array}{l}\text { Refiere a la intención y a } \\
\text { la capacidad de la entidad } \\
\text { de mantener el } \\
\text { instrumento financiero, ya } \\
\text { sea hasta su vencimiento } \\
\text { o para su negociación a } \\
\text { corto plazo. }\end{array}$ & $\begin{array}{l}\text { La clasificación se } \\
\text { encuentra basada en un } \\
\text { modelo de negocio y } \\
\text { las características de los } \\
\text { flujos de caja } \\
\text { contractuales de cada } \\
\text { instrumento financiero. }\end{array}$ \\
\hline
\end{tabular}

Inversiones disponibles para la venta
Es una categoría residual, Ya no existe. Sin pues no son ni mantenidos para su negociación ni a vencimiento. No tiene restricción. embargo, su medición (a valor razonable con cambios en otro resultado integral) se aplica cuando se cumple uno de los modelos de negocio para instrumentos de deuda y de patrimonio. 


\begin{tabular}{|c|c|c|c|}
\hline $\begin{array}{l}\text { Instrumentos de } \\
\text { deuda }\end{array}$ & $\begin{array}{l}\text { Forma de medición } \\
\text { posterior }\end{array}$ & $\begin{array}{l}\text { Costo amortizado } \\
\text { para instrumentos } \\
\text { mantenidos hasta el } \\
\text { vencimiento A valor } \\
\text { razonable con } \\
\text { cambios en } \\
\text { resultados. } \\
\text { A valor razonable } \\
\text { con cambios en } \\
\text { otros resultados } \\
\text { integrales para } \\
\text { inversiones } \\
\text { disponibles para la } \\
\text { venta. }\end{array}$ & $\begin{array}{l}\text { Costo amortizado si el objeto del } \\
\text { negocio es cobrar los flujos de caja } \\
\text { contractuales que son el principal e } \\
\text { intereses sobre el instrumento } \\
\text { A valor razonable con cambios en } \\
\text { otro resultado integral si el objetivo } \\
\text { del modelo de negocio consiste en la } \\
\text { obtención de los flujos de caja } \\
\text { contractuales del instrumento (deben } \\
\text { ser únicamente por el pago del } \\
\text { principal e intereses sobre el mismo) y } \\
\text { también para vender los activos } \\
\text { A valor razonable con cambios en } \\
\text { resultados si el modelo de negocio no } \\
\text { cumple con ninguna de las } \\
\text { características antes mencionadas y si } \\
\text { los flujos de caja contractuales no } \\
\text { pasan la prueba de SPPI. } \\
\text { A valor razonable con cambios en } \\
\text { resultados con el fundamento de que, } \\
\text { con ello, se elimina una asimetría } \\
\text { contable. }\end{array}$ \\
\hline & Reclasificaciones & $\begin{array}{l}\text { Están permitidas en } \\
\text { circunstancias } \\
\text { específicas, y las } \\
\text { ganancias o } \\
\text { pérdidas son } \\
\text { tratadas de manera } \\
\text { diferente } \\
\text { dependiendo del } \\
\text { movimiento entre } \\
\text { las categoría. }\end{array}$ & $\begin{array}{l}\text { Están permitidas a partir del cambio } \\
\text { en el modelo de negocio, a nivel del } \\
\text { total de los instrumentos o portafolios } \\
\text { afectados, los cuales deben estar } \\
\text { sustentados, aprobados por la } \\
\text { Gerencia y deben ser poco frecuentes } \\
\text { (pues el modelo de negocio es } \\
\text { estable). }\end{array}$ \\
\hline
\end{tabular}




\begin{tabular}{lll}
\hline $\begin{array}{l}\text { Instrumento } \\
\text { de }\end{array}$ patrimonio & $\begin{array}{l}\text { Forma de } \\
\text { medición } \\
\text { posterior }\end{array}$ & $\begin{array}{l}\text { A valore razonable con cambios } \\
\text { en resultados si son mantenidos } \\
\text { para negociación. }\end{array}$ \\
& & A valor razonable con cambios \\
en otro resultado integral para \\
la clasificación de inversiones \\
disponibles para la venta. Las \\
ganancias y/o pérdidas \\
& & registradas en los resultados no \\
& realizados (cuenta patrimonial) \\
& se reclasifican a resultados del \\
período. & \\
& Si existen inversiones que no \\
& cotizan en un mercado activo, \\
& se podrían medir \\
& excepcionalmente al costo \\
& cuando el valor razonable no \\
& pueda ser determinado con \\
& confiabilidad.
\end{tabular}

Reclasificaciones Entre las categorías de valor razonable con cambios en resultados y de disponible para la venta, las reclasificaciones están permitidas. Cuando se transfieren desde disponibles para la venta, los resultados no realizados se reconocen en los resultados del ejercicio. Cuando se transfieren desde la categoría de valor razonable con cambios en resultados, no está permitido realizar ninguna reversión de las ganancias o pérdidas realizadas a no realizadas.
A valor razonable con cambios en resultado.

Opción sin cambio de designar el instrumento en el momento inicial como a valor razonable con cambios en otro resultado integral con la condición de que el instrumento no sea mantenido para negociación. Esta opción es llamada como «sin reciclaje», pues los resultados generados no se reconocerán en los resultados del período en el caso de su enajenación y/o baja, sino seguirán en otra cuenta patrimonial. Los dividendos sí se registran en los resultados del ejercicio.

Se elimina la posibilidad de medir al costo inversiones de patrimonio no cotizadas cuando el valor razonable no pueda ser medido confiablemente.

Entre las categorías de a valor razonable con cambios en resultados hacia valor razonable con cambios en otros resultados integrales, las reclasificaciones no están permitidas, debido a que la elección de llevarlo a valor razonable con cambios en otros resultados integrales se realiza en el reconocimiento inicial del instrumento de manera irrevocable.

Fuente: (Rodríguez, 2017) 
De forma resumida la autora Silvestre (2017) indica que los cambios entre la NIC 39 y la NIIF 9 se basan fundamentalmente en tres aspectos:

- Nueva clasificación, a efectos de valoración, de los activos y pasivos financieros

- Nueva metodología para el cálculo del deterioro de valor de activos financieros

- Simplificación de la contabilidad de coberturas

\section{Medición de los instrumentos financieros}

- Un activo financiero o instrumento financiero deberá medirse según indica Hernández (2017), de esta manera:

- A costo amortizado: si está dentro de un modelo de negocio que tiene como objetivo obtener los flujos de efectivo contractuales del activo, y si las condiciones de éste dan lugar a flujos de efectivo que son únicamente pagos del principal e intereses sobre el importe del principal pendiente.

- A valor razonable con cambios en otro resultado integral: si el objetivo del modelo de negocio en el que se mantiene el activo financiero es obtener sus flujos contractuales, pero tener la posibilidad de venderlo, y si las condiciones contractuales del activo dan lugar a flujos de efectivo que son únicamente pagos del principal e intereses sobre el importe del principal pendiente.

- A valor razonable con cambios en resultados: por diferencia de los anteriores, pero de acuerdo con los criterios ya mencionados.
De igual manera, Medina (2018) menciona que, como regla general los activos y los pasivos financieros se deben medir a valor razonable, si no se contabiliza a valor razonable incluyendo los cambios en los resultados, se le debe agregar los costos de transacción que sean atribuibles al momento de su adquisición o su emisión.

Una excepción presente en la medición son aquellas cuentas comerciales a cobrar que no posean un componente financiero considerable, en este sentido se deben valorar según el precio de su transacción.

\section{Clasificación y medición posterior de activos financieros}

Para este caso Medina (2018) menciona que la NIIF 9 posee un nuevo enfoque de clasificación tomando en consideración dos aspectos:

- Las características de los flujos de efectivo contractuales de los activos

- Modelo de negocio de la entidad

- Por tanto antes de que la empresa realice la clasificación de los instrumentos financieros deberá aplicar algunas comprobaciones:

- $\quad$ Test de modelo de negocio.

- Test de los flujos contractuales

- En este sentido y de acuerdo a estos dos aspectos antes mencionados la NIIF en su Capítulo 4 determina los tipos de medición posterior:

- A coste amortizado

- Valor razonable con cambios en patrimonio neto 
- Valor razonable con cambios en resultados.
En base a estos aspectos se puede seleccionar el tipo de valoración que se requiere para cada caso y se muestran en la Tabla 3.

Tabla 1

Aspectos a considerar para seleccionar el tipo de valoración a aplicar

\section{ASPECTO A CONSIDERAR}

Un activo financiero se medirá a coste amortizado cuando se cumplan dos requisitos.

\section{REQUISITOS QUE DEBE CUMPLIR}

El modelo de negocio persigue mantener el activo financiero para obtener flujos de efectivo contractuales.

Las condiciones del contrato del activo financiero dan lugar a flujos de efectivo en fechas especificadas que son únicamente pagos del principal e intereses sobre el importe pendiente del principal.

Un activo financiero debe medirse a valor razonable El modelo de negocio tiene como objetivo la con cambios en otro resultado integral cuando se cumplan dos requisitos. obtención de flujos de efectivo contractuales y vender activos financieros - Las condiciones del contrato del activo financiero dan lugar a flujos de efectivo en fechas especificadas que son únicamente pagos del principal e intereses sobre el importe pendiente del principal.

Un activo financiero debe medirse a valor razonable Si el modelo de negocio en el que se encuentra no con cambios en resultados. está sujeto a otro modelo de negocio.

Fuente: (Medina, 2018)

A pesar de que la norma está dirigida principalmente a entidades bancarias y empresas aseguradoras, es preciso que las organizaciones no financieras estudien los beneficios que esta norma trae a su empresa y de hecho se podría aplicar de manera más sencilla puesto que sus instrumentos financieros son más reducidos como las cuentas por cobrar comerciales y depósitos bancarios en comparación a las empresas aseguradoras cuyo proceso de medición es más complejo.

\section{CONCLUSIONES}

Las Normas Internacionales de Información financiera, son fundamentales para lograr una mayor transparencia en la presentación de los estados financieros y en el caso más específico la 
NIIF 9 permite estudiar los instrumentos financieros, medirlos y evaluarlos teniendo una mayor claridad del comportamiento de cada uno de los instrumentos financieros lo que permite tomar decisiones más precisas.

Es evidente que la NIF 9 es compleja, sin embargo puede ser analizada y comprendida para la aplicación de su fundamentación logrando tener un impacto en el análisis de los instrumentos financieros y su medición. Cada uno de ellos cumple una función específica que debe tenerse muy clara para evitar errores al momento de reflejar los estados financieros, logrando un mejor desenvolvimiento de las empresas asegurando la permanencia en el mercado por mucho tiempo.

La NIIF 9 al igual que el resto de las normas se pueden ir adaptando de acuerdo a los cambios producto de la globalización y las exigencias del mercado, para mantener un equilibrio económico en cada una de las empresas que deban aplicar estas normas y así evitar sucumbir en errores que sean desencadenantes de malos procedimientos financieros y comerciales. 


\section{REFERENCIAS BIBLIOGRÁFICAS}

Buján, A. (17 de Abril de 2018). Instrumentos Financieros. Recuperado el 29 de Junio de 2018, de https:// www.enciclopediafi nanciera.com/ instrumentos-financieros.htm

Durán, S., Crissien, J., Virviescas, J., \& García, J. (2017). Estrategías gerenciales para la formación de equipos de trabajos en empresas constructoras del caribe Colombiano. Revista Espacios , 38 (13).

Enríquez, E. (12 de Noviembre de 2019). Normativa Vigente NIIF. Recuperado el 20 de Agosto de 2021, de https://www.ifs-group.ec/post/ normativa-vigente-niif

Filgueira, C. (2017). Manual Contable NIIF: aplicación práctica. Recuperado el 20 de Agosto de 2021, de https://books.google.es/ books?hl=es\&lr=\&id=20FYDwAAQBAJ\&oi $=$ fnd $\& p g=$ PT62\&dq $=$ Manual + Contable $+\mathrm{NI}$ IF:+aplicaci\%C3\%B3n+pr\%C3\%A1ctica\&ot $\mathrm{s}=\mathrm{t}$ e $\mathrm{M} \mathrm{K} \mathrm{m} \mathrm{h} \mathrm{h} 0 \mathrm{zK} \& \mathrm{sig}=$ ReatpobdPrUiODSs5y-3HvPUVI

Goyburo, A. (2018). Análisiis en la implementación de la NIIF 9 "Instrumentos financieros" en entidades no financieras. Samborondón: Trabajo especial de grado de la Universidad de Especialidades Espírtu Santo para optar al título de Contador Público Autorizado.

Hernández, C. (15 de Diciembre de 2017). Recuperado el 21 de Agosto de 2021, de Recuerde que a partir del 1 de enero de 2018 entrará en vigencia la NIIF 9: https://www.incp.org.co/ recuerde-partir-del-1-enero-2018-entraravigencia-la-niif-9/
Medina, I. (2018). Hacia una nueva era: Norma Internacional de Información Financiera 9. Madrid: Ensayo de la Universidad Pontificia Comillas.

Morales-Díaz, J. (2018). La última reforma de las NIIF y sus efectos en los estados financieros. Estudios de economia aplicada 36(2), 349-378.

Ortegón, N. (2017). La NIIF 9 los instrumentos financieros dentro del proceso contable: análisis sobre la norma internacional de información financiera 9. Bogotá, Colombia: Informe de la Universidad Militar Nueva Granada.

Palella Stracuzzi, S., \& Martins Pestana, F. (2010). Metodología de la investigación cuantitativa. Caracas, Venezuela: FEDUPEL, Fondo Editorial de la Universidad Pedagógica Experimental Libertador.

Parrales, C., \& Castillo, F. (2018). Análisis NIIF 9Instrumentos Fiinancieros desde una perspectiva Industrial. Actualidad Contable. Contabilidad y negocios 13(25), 6-19.

Rodríguez, D. (2017). Antes NIC 39 ahora NIIF 9: Nuevos desafios para los contadores. Contabilidad y Negocios: Revista de departamento académico de ciencias administrativas 12 (23) , 6-21.

Rodríguez, D. (26 de Septiembre de 2015). Normas Internacionales de Información Financiera (NIIF). Recuperado el 19 de Agosto de 2021, de https://contabilidad.com.do/niif/

Silvestre, M. (9 de Octubre de 2017). LA NUEVA 
$\begin{array}{llllllll}R & \text { E } & \text { V } & \text { I } & S & T & A\end{array}$

Public a n d o

I S S N $13390-9304$

CONTABILIDAD DE LOS

INSTRUMENTOS FINANCIEROS. (A.

Internacional, Editor) Recuperado el 20 de

Agosto de 2021, de https://

www.auren.com/es-ES/blog/auditoria/

2017-10-18/la-nueva-contabilidad-de-los-

instrumentos-financieros 